\title{
Deficiency of haematopoietic-cell-derived IL-10 does not exacerbate high-fat-diet-induced inflammation or insulin resistance in mice
}

\author{
G. M. Kowalski • H. T. Nicholls • S. Risis • \\ N. K. Watson • P. Kanellakis • C. R. Bruce • A. Bobik • \\ G. I. Lancaster • M. A. Febbraio
}

Received: 27 May 2010 / Accepted: 25 November 2010 / Published online: 6 January 2011

(C) Springer-Verlag 2011

\begin{abstract}
Aims/hypothesis Recent work has identified the important roles of M1 pro-inflammatory and M2 anti-inflammatory macrophages in the regulation of insulin sensitivity. Specifically, increased numbers of M2 macrophages and a decrease in M1 macrophages within the adipose tissue are associated with a state of enhanced insulin sensitivity. IL-10 is an anti-inflammatory cytokine and is a critical effector molecule of M2 macrophages.

Methods In the present study, we examined the contribution of haematopoietic-cell-derived IL-10 to the development of obesity-induced inflammation and insulin resistance. We
\end{abstract}

Electronic supplementary material The online version of this article (doi:10.1007/s00125-010-2020-5) contains supplementary material, which is available to authorised users.

G. M. Kowalski $\cdot$ H. T. Nicholls $\cdot$ S. Risis $\cdot$ N. K. Watson •

C. R. Bruce $\cdot$ G. I. Lancaster $(\bowtie) \cdot$ M. A. Febbraio $(\bowtie)$

Cellular \& Molecular Metabolism Laboratory,

Baker IDI Heart \& Diabetes Institute,

PO Box 6492, St Kilda Road Central,

Melbourne, 3008 VIC, Australia

e-mail: graeme.lancaster@bakeridi.edu.au

M. A. Febbraio

e-mail: mark.febbraio@bakeridi.edu.au

G. M. Kowalski $\cdot$ H. T. Nicholls $\cdot$ M. A. Febbraio

Department of Biochemistry and Molecular Biology,

Monash University,

Clayton,

Melbourne, VIC, Australia

P. Kanellakis $\cdot$ A. Bobik

Vascular Biology \& Atherosclerosis Laboratory,

Baker IDI Heart \& Diabetes Institute,

Melbourne, VIC, Australia hypothesised that haematopoietic-cell-restricted deletion of IL-10 would exacerbate obesity-induced inflammation and insulin resistance. Lethally irradiated wild-type recipient mice receiving bone marrow from either wild-type or $1 l 10$-knockout mice were placed on either a chow or a high-fat diet for a period of 12 weeks and assessed for alterations in body composition, tissue inflammation and glucose and insulin tolerance.

Results Contrary to our hypothesis, neither inflammation, as measured by the activation of pro-inflammatory stress kinases and gene expression of several pro-inflammatory cytokines in the adipose tissue and liver, nor diet-induced obesity and insulin resistance were exacerbated by the deletion of haematopoietic-cell-derived IL-10. Interestingly, however, Ill0 mRNA expression and IL-10 protein production in liver and/or adipose tissue were markedly elevated in Il10-knockout bone-marrow-transplanted mice relative to wild-type bone marrow-transplanted mice.

Conclusions/interpretation These data show that deletion of IL-10 from the haematopoietic system does not potentiate high-fat diet-induced inflammation or insulin resistance.

Keywords Anti-inflammatory cytokines · Glucose tolerance $\cdot$ Macrophage polarisation $\cdot$ Obesity

$\begin{array}{ll}\text { Abbreviations } \\ \text { AMREP } & \begin{array}{l}\text { Alfred Medical Research and Education } \\ \text { Precinct }\end{array} \\ \text { ATM } & \text { Adipose tissue macrophage } \\ \text { BMDM } & \begin{array}{l}\text { Bone-marrow-derived macrophage } \\ \text { BMT }\end{array} \\ \text { Bone marrow transplant } \\ \text { CLAMS } & \begin{array}{l}\text { Comprehensive Laboratory Animal Monitoring } \\ \text { System }\end{array} \\ \text { IKK } \beta & \text { Inhibitor of kappa kinase } \beta\end{array}$

Abbreviations

Inhibitor of kappa kinase $\beta$ 
IPGTT Intraperitoneal glucose tolerance test

IPITT Intraperitoneal insulin tolerance test

JNK c-Jun N-terminal kinase

LBM Lean body mass

LPS Lipopolysaccharide

MGL1 Macrophage galactose $N$-acetyl-galactosaminespecific lectin 1

PPAR Peroxisome proliferator-activated receptor

RER Respiratory exchange ratio

$\dot{V} \mathrm{O}_{2} \quad$ Whole-body oxygen uptake

\section{Introduction}

Obesity, insulin resistance and type 2 diabetes are closely associated with a state of 'low-grade' chronic inflammation [1]. Specifically, elevated levels of pro-inflammatory cytokines such as TNF $\alpha$, and infiltration of bone-marrow-derived macrophages (BMDMs) into adipose tissue appear to be key events mediating the deleterious effects of obesity on insulin sensitivity [2-4]. It is generally accepted that macrophages accumulate in the adipose tissue of obese humans and mice. Once resident within the adipose tissue, factors secreted by macrophages, such as TNF $\alpha$, activate pro-inflammatory signalling molecules, such as c-Jun $\mathrm{N}$-terminal kinase (JNK) and $\operatorname{I\kappa B}$ kinase $\beta(\operatorname{IKK} \beta)$, and initiate a state of local and, ultimately, systemic insulin resistance [5-8]. Underscoring the critical role of macrophages in the development of obesity-induced insulin resistance, the conditional deletion of $\mathrm{CD} 11 \mathrm{c}^{+}$-expressing macrophages in obese mice results in a marked reduction in local and systemic inflammation and, importantly, normalisation of insulin sensitivity [9].

Resident tissue macrophages display considerable heterogeneity [10]. Lean chow-fed animals contain small amounts of resident adipose tissue macrophages (ATMs) and these ATMs stain positive for the surface markers F480, CD11b, and the anti-inflammatory marker macrophage galactose $N$-acetyl-galactosamine-specific lectin 1 (MGL1/CD301), but stain negative for CD11c. These $\mathrm{MGL}^{+} \mathrm{CD} 11 \mathrm{c}^{-}$ATM found in lean animals express many genes typical of the M2 alternatively activated state, including high expression of 1110 , encoding the anti-inflammatory cytokine IL-10 [11-13]. In contrast, in high-fat-fed and genetically obese mice, macrophages show a characteristic triple-positive staining for F4/80, CD11b and CD11c and an absence of staining for MGL1/ CD301 [11, 12, 14]. Thus, the progression from the lean to obese state results in a reduced expression of antiinflammatory M2-macrophage-related genes, namely Argl and Ill0, with a concomitant increase in the expression of pro-inflammatory M1-macrophage genes, including Tnf- $\alpha$ (also known as Tnf) and iNos (also known as Nos2), in ATM [11, 12]. While high-fat feeding does not alter the presence of the $\mathrm{M} 2 \mathrm{MGL1}^{+} \mathrm{CD} 11 \mathrm{c}^{-}$ cells, it induces the spatially restricted and more rapid accumulation of pro-inflammatory $\mathrm{M} 1 \mathrm{MGL1}^{-} \mathrm{CD} 11 \mathrm{c}^{+}$ ATMs in 'crown-like clusters' surrounding dead adipocytes in the visceral region [12]. While the above studies support the idea that high-fat-feeding-induced obesity promotes an M2 to M1 polarisation shift, more recent data suggest that the M1-M2 ATM profile is relatively dynamic in nature and over the course of the high-fat-dietary period the M1 to M2 macrophage balance shifts, potentially reflecting periods of adipose tissue hypertrophy and re-modelling [15]. Underscoring the importance of macrophage polarisation state to the development of obesity-induced insulin resistance, the insulin-sensitising agent rosiglitazone dramatically increases the number of macrophages present in the adipose tissue depots of obese mice; however, these ATM are predominantly of the M2 anti-inflammatory state [16]. Recently, peroxisome proliferator-activated receptor $\gamma$ $(\operatorname{PPAR} \gamma)$ and $\delta$ (PPAR $\delta)$ were identified as critical promoters of macrophage polarisation towards the M2 phenotype and, consequently, macrophage-specific deletion of PPAR $\gamma$ or PPAR $\delta$ resulted in a reduced expression of M2macrophage-specific genes in adipose tissue and liver, an increase in M1-macrophage-specific genes and, importantly, a worsening of glucose and insulin tolerance $[17,18]$.

While M2 macrophages express a wide range of antiinflammatory genes, a critical effector of M2 macrophages is the anti-inflammatory cytokine IL-10 [19, 20]. Indeed, IL-10 or IL-10 receptor $\beta$-chain deficient mice develop chronic enterocolitis, an effect that can be substantially prevented with the treatment of IL-10 in Il10-knockout (KO) mice, demonstrating the importance of this cytokine in the regulation of host inflammatory responses [21-23]. Most [11, 24-28] but not all [29, 30] studies, support a protective role of IL-10 in the regulation of metabolic inflammation and insulin sensitivity both in vitro and in vivo. In addition, $I l 10$ expression increases in adipose tissue following weight loss in obese patients, concomitant with a reduction in proinflammatory gene expression $[31,32]$. To determine the contribution of haematopoietic-cell-derived IL-10 to the development of obesity-induced inflammation and insulin resistance we have used a bone marrow adoptive transfer model and hypothesised that haematopoietic-cell-restricted deletion of IL-10 would potentiate high-fat-diet-induced inflammation and insulin resistance.

\section{Methods}

Animal procedures Wild-type C57BL/6 and global Il10$\mathrm{KO}$ mice bred onto a C57BL/6 background (Jackson Laboratory, Bar Harbor, ME, USA) were housed in 
pathogen-free barrier-protected environment (12:12 h light/ dark cycle) at the Baker IDI Alfred Medical Research and Education Precinct (AMREP) animal facility. All procedures were approved by the AMREP Animal Ethics Committee. When the mice were 6 weeks of age, we performed the bone marrow transplant (BMT) experiment described in detail in the Electronic supplementary material (ESM).

Body composition and metabolic data analysis Over the entire course of the dietary intervention, lean body mass (LBM) and fat mass were measured fortnightly in conscious mice using the four in one EcoMRI body composition analyser (Columbus Instruments, Columbus, $\mathrm{OH}$, USA). Mouse metabolic data were also collected at week 12 using the Comprehensive Laboratory Animal Monitoring System (CLAMS, Columbus Instruments). Mice were placed inside the metabolism cages for a 26-h period, with the first $2 \mathrm{~h}$ of data not included to allow for acclimatisation of mice to the cages. Oxygen-consumption and food-intake data were normalised to total body mass $(\mathrm{ml} / \mathrm{h}$ and $\mathrm{kJ} / \mathrm{day}$, respectively).

Glucose homeostasis data Intraperitoneal glucose tolerance tests (IPGTT) and intraperitoneal insulin tolerance tests (IPITT) were performed at the end of the 12 week dietary intervention following a $6 \mathrm{~h}$ fast. Mice received an intraperitoneal injection of $1 \mathrm{~g}$ glucose $/ \mathrm{kg} \mathrm{LBM}$ and $0.6 \mathrm{U} / \mathrm{kg} \mathrm{LBM}$ (chow) or $0.75 \mathrm{U} / \mathrm{kg} \mathrm{LBM}$ (high-fat diet) human insulin (Humulin, Eli Lilly, IN, USA) during the IPGTT and IPITT, respectively. By using a lower dose (0.6 U/kg LBM) of insulin in the chow-fed animals, incidents of extreme hypoglycaemia were avoided. Tail blood was collected over the $120 \mathrm{~min}$ of the IPGTT and IPITT and glucose levels were measured with human Accu-Chek blood monitors and test strips (Roche, Basel, Switzerland). Fasting and fed plasma insulin concentrations were analysed using rat/mouse insulin ELISA kits (Millipore, Billerica, MA, USA).

Cell culture RAW264.7 murine macrophages were cultured in Dulbecco's modified Eagle's medium (Invitrogen, Carlsbad, CA, USA) containing low glucose and supplemented with $10 \%$ FBS and $1 \%$ penicillin/streptomycin (Invitrogen). The cells were grown to $\sim 70-80 \%$ confluence and then treated with $0.5 \mathrm{mmol} / \mathrm{l}$ palmitate (Sigma-Aldrich, St Louis, MO, USA) conjugated to $2 \%$ BSA (wt/vol.) for $4 \mathrm{~h}$, either in the presence or absence of $20 \mathrm{ng} / \mathrm{ml}$ recombinant murine IL-10 (PeproTech, Rocky Hill, NJ, USA). The control medium was also supplemented with $2 \%$ BSA. Additionally, BMDMs from wild-type C57BL/6 control and global $I l 10-\mathrm{KO}$ mice were also treated with $100 \mathrm{ng} / \mathrm{ml}$ lipopolysaccharide (LPS) (L8274, Sigma-Aldrich), $20 \mathrm{ng} / \mathrm{ml}$ recombinant murine IL-4 (R\&D Systems, St Louis, MO, USA) and 0.5 and $0.75 \mathrm{mmol} / 1$ palmitate (Sigma-Aldrich) for the times indicated. Following treatment, medium supernatant fractions were collected, cells were washed twice with ice-cold PBS and scraped in ice-cold lysis buffer or TRIzol Reagent (Invitrogen) and stored at $-79^{\circ} \mathrm{C}$.

BMDM experiments To obtain primary BMDMs, mice between 6 and 12 weeks of age were killed with $\mathrm{CO}_{2}$ asphyxiation. Hind leg bones were stripped of muscle and connective tissues, placed in ethanol for $1 \mathrm{~min}$ and then icecold PBS. Marrow was flushed out with RPMI 1640 medium and spun at $500 \mathrm{~g}$ for $10 \mathrm{~min}$. Cells were seeded $1 \times 10^{6} / \mathrm{ml}$ in RPMI 1640 media (Invitrogen) supplemented with glutamine and Hepes, 20\% (vol./vol.) conditioned media from L929 cells, 15\% FBS (vol./vol.), $100 \mathrm{U} / \mathrm{ml}$ penicillin and $0.1 \mathrm{mg} / \mathrm{ml}$ streptomycin. Cells remaining in suspension $24 \mathrm{~h}$ later were divided into treatment plates with additional supplemented RPMI medium and differentiated into macrophages over 4-7 days.

Protein analyses Proteins were analysed by immunohistochemistry and western blot analyses using standard techniques described in detail in the ESM.

Real-time PCR To extract RNA, $\sim 30-50 \mathrm{mg}$ of either epididymal white adipose tissue or liver was homogenised in $800 \mu \mathrm{l}$ of TRIzol Reagent according to the manufacturer's instructions (Invitrogen). Total RNA quantity and quality was measured using the ND-1000 NanoDrop Spectrophotometer (Thermo Scientific, Waltham, MA, USA). For each sample, 1,500 ng RNA was reverse transcribed and converted to cDNA using TaqMan Reverse Transcription Reagents (Applied Biosystems, Foster City, CA, USA). All gene expression analysis was performed using TaqMan Gene Expression Assays (Applied Biosystems), including $18 \mathrm{~S}$ probe and primers for housekeeping gene measurement. In each RT-PCR reaction, $50 \mathrm{ng}$ of total cDNA was used.

Statistical analysis Data were analysed with either two-way ANOVA or three-way repeated-measures ANOVA (SigmaStat Version 3.5) where appropriate. All data are expressed as means \pm SEM. A $p$ value of less than $<0.05$ was used to denote statistical significance.

\section{Results}

Elevated levels of fatty acids, in particular long-chain saturated fatty acids, are postulated to be key drivers of inflammation. Therefore, we initially examined whether IL-10 could prevent 
palmitate-induced inflammation. Accordingly, we treated Raw264.7 macrophages with $0.5 \mathrm{mmol} / 1$ palmitate for $4 \mathrm{~h}$, either in the presence or absence of IL-10. As shown previously by Shi et al. [33], palmitate increased both membrane-bound and secreted TNF- $\alpha$ (Fig. 1a, b), but co-treatment with IL-10 markedly reduced palmitate-induced increases in both membrane-bound and secreted TNF- $\alpha$ (Fig. 1a, b).

Given that IL-10 treatment prevented the palmitateinduced secretion of TNF- $\alpha$ in macrophages in vitro, we investigated the role of immune-cell-derived IL-10 in the development of high-fat-diet-induced obesity, inflammation and insulin resistance in vivo. To this end, we transplanted lethally irradiated mice as described above. Consistent with previously published reports [8, 34, 35], bone marrow was reconstituted with $\sim 95 \%$ efficiency (Fig. 2). While a small amount of endogenous recipient-derived white blood cells

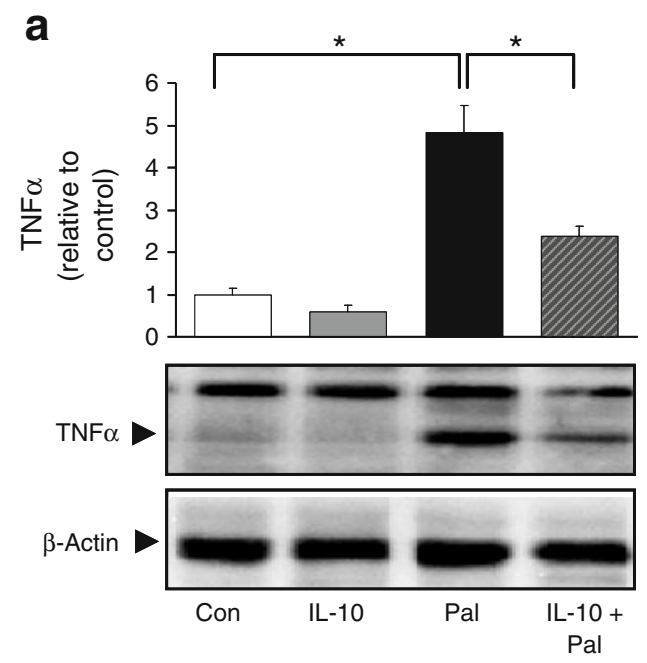

b

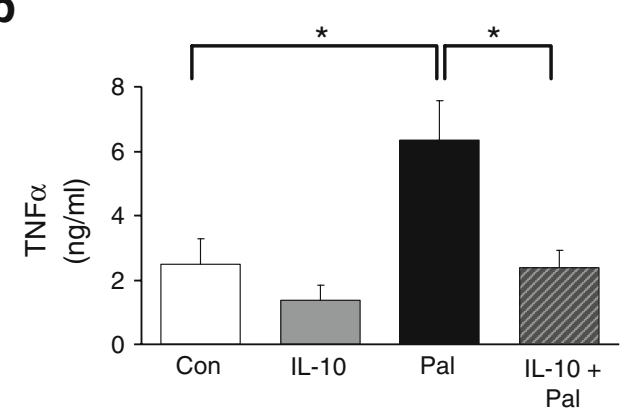

Fig. 1 IL-10 suppresses lipid-induced TNF- $\alpha$ production in Raw264.7 macrophages. a,b Raw264.7 macrophages treated for $4 \mathrm{~h}$ with: BSA-containing control media (Con); recombinant mouse IL-10 (20 ng/ml) (IL-10); or $0.5 \mathrm{mmol} / 1$ palmitate (2\% BSA; Pal); or co-treated with IL-10 and palmitate (IL-10+Pal). a Western blot analysis of pro-TNF- $\alpha$ (membrane bound) with densitometry quantification from cell lysates. $\mathbf{b}$ Analysis of secreted TNF- $\alpha$ from cell culture media supernatant fractions as measured by ELISA. Representation of three and two independent experiments performed in triplicate, respectively. Data are means $\pm \mathrm{SEM}$; interaction effect, $* p<0.05$

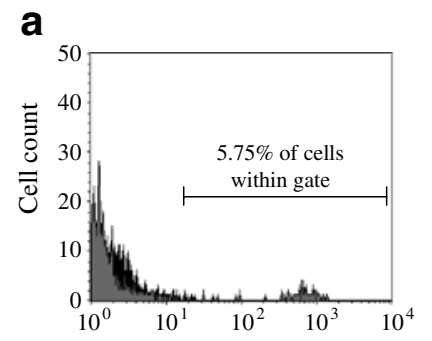

\section{b}

C
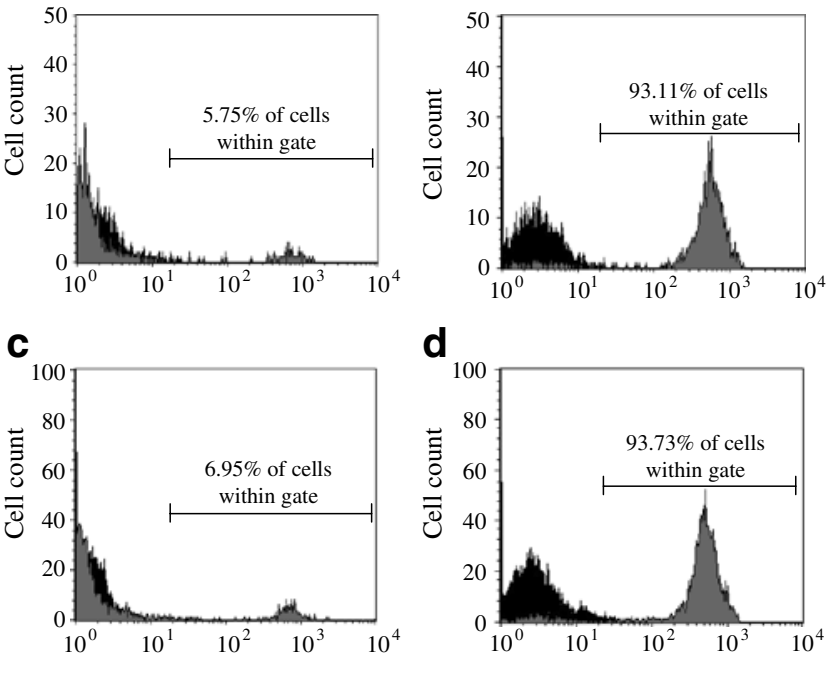

d

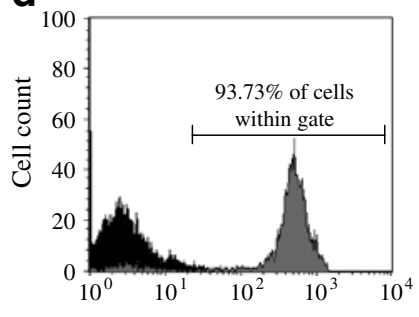

e

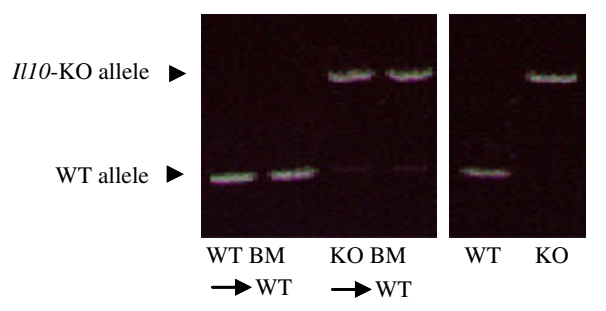

Fig. 2 BMT results in successful repopulation of WT and Ill0-KO haematopoietic cells in lethally irradiated mice. a-d Male 6-week-old C57BL/6 mice were lethally irradiated, then tail-vein injected with bone marrow from donor wild-type $(\mathbf{a}, \mathbf{b})$ or global $1110-\mathrm{KO}(\mathbf{c}, \mathbf{d})$ mice. Following 6 weeks' recovery, bone marrow reconstitution efficiency was measured using congenic donor and recipient mice that differed at the Ly5.1/Ly5.2 leucocyte locus. a, c Whole blood was collected and stained with anti-CD45.1-PE (Ly5.1) antibodies (grey) or isotype controls (black) and analysed by flow cytometry, revealing $5.75 \%$ (a) and $6.95 \%$ (c) of remnant endogenous leucocytes following transplantation with WT (a) and Ill0-KO (c) bone marrow cells, respectively. b, d Whole blood stained with anti-CD45.2-FITC (Ly5.2) antibodies (grey) or isotype controls (black), showing 93.11\% (b) and 93.73\% (d) of leucocytes are of donor WT (b) or Il10-KO (d) origin, respectively. e Following the end of the dietary period (18 weeks following BMT) whole blood was collected from mice and DNA PCR genotyped for the presence or absence of the wild-type or mutant $I l 10$ allele. Results from two representative mice from each genotype are shown. Wild-type and global Ill0-KO tail DNA was used as a positive control. BM, bone marrow; $\mathrm{KO}$, knockout; WT, wild type

remained in the circulation of the transplanted animals, no differences in reconstitution efficiency were observed between mice receiving wild-type or $1 l 10-\mathrm{KO}$ bone marrow (Fig. 2). Additionally, to confirm transplantation efficacy for each animal, whole blood was collected from each transplanted animal at the end of the study (18 weeks) and PCR genotyped for the wild-type or mutant $1110-\mathrm{KO}$ allele. High levels of repopulation with donor white blood cells were observed in all animals (Fig. 2e). Wild-type and global Ill0-KO mouse tissue serves here as the positive 
control (Fig. 2e). It is well established that the BMT technique results in very efficient repopulation of donor myeloid cells not only into the circulation, but into tissues such as the liver and adipose tissue after high-fat feeding [8]. Indeed, it has been demonstrated that most of the F4/ $80^{+}$cells recruited to white adipose tissue in response to high-fat feeding are bone marrow derived [3]. Thus, the BMT technique is an efficient method to replace both circulating and tissue-resident bone-marrow-derived cells. Notwithstanding this, in the current study we did not determine tissue specific repopulation by donor bone marrow cells. Therefore, while it is likely that the majority of cells recruited to the white adipose tissue in response to high-fat feeding are donor bone marrow derived, we cannot categorically determine this in the current study.

Six weeks after recovery from the BMT procedure mice were randomised to chow- or high-fat-diet groups. As expected, mice placed on the high-fat diet accumulated more body fat than those on the chow diet (Fig. 3). No differences in body weight or fat mass were observed between animals receiving wild-type or IL-10 bone
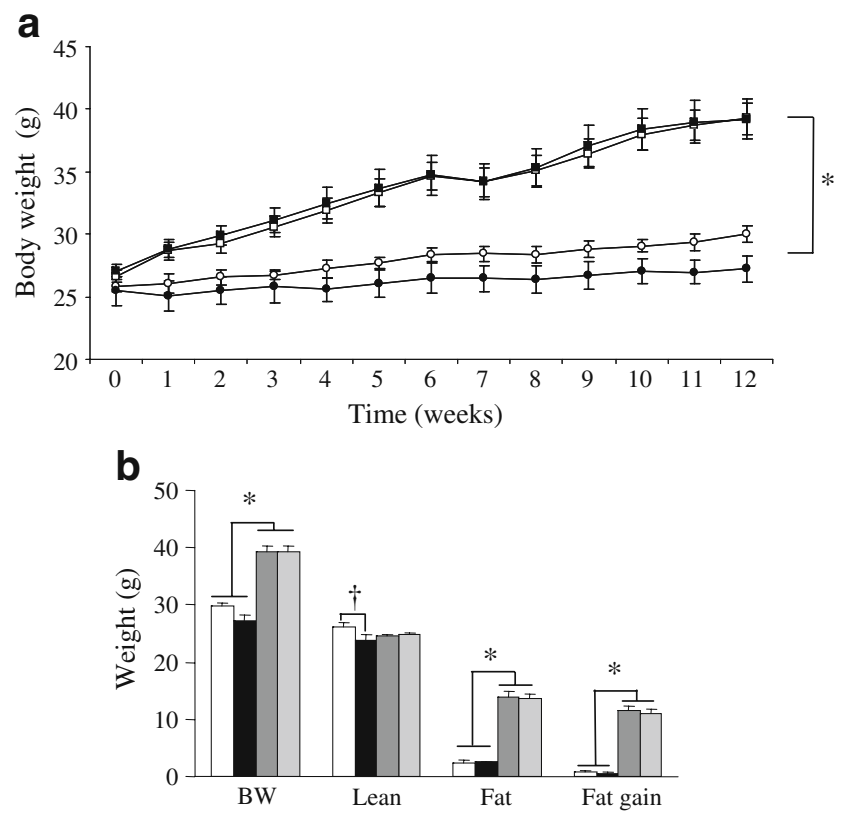

Fig. 3 Ill0-KO BMT mice on both chow and high-fat diets have no differences in body weight or fat mass compared with wild-type BMT controls. Mice were routinely weighed and body composition assessed using EcoMRI throughout the entire dietary period. a Change in body weight over time. Circles, chow-fed mice; squares, high-fat-fed mice; white symbols, wild-type BMT mice; black symbols, Il10-KO BMT mice. b Body composition data at the end of week 12. White bars, chow-fed wild-type BMT mice; black bars, chow-fed Ill0-KO BMT mice; dark grey bars, high-fat-fed wild-type BMT mice; light grey bars, high-fat-fed Il10-KO BMT mice. Data are means \pm SEM. Main effect for diet, ${ }^{*} p<0.05$; diet-genotype interaction effect, ${ }^{\dagger} p<0$.05. Chow, $n=5-6$ mice per genotype; high-fat diet, $n=11-16$ per genotype. BW, body weight marrow (Fig. 3). There was a small but significant genotype-diet interaction for LBM $(p<0.05)$, with chow-fed Ill0-KO BMT mice having less LBM than the chow-fed wild-type BMT mice at the end of the dietary period (Fig. 3). This effect likely reflects the lower lean mass in this group of mice at the commencement of the study. There were no differences in LBM between wildtype BMT and $1 l 10-\mathrm{KO}$ BMT mice on the high-fat diet (Fig. 3). These data demonstrate that deletion of IL-10 within the haematopoietic compartment does not affect fat mass accumulation with either the chow or the high-fat diet.

In addition to measuring body composition, metabolic data were collected using the CLAMS apparatus (Table 1). There were no differences in food intake, respiratory exchange ratio (RER) or physical activity between Il10KO BMT and wild-type BMT mice on either the chow or high-fat diet (Table 1). As expected there was a strong dietary effect for RER, in that mice on the high-fat diet had a markedly lower RER, indicating a shift in substrate utilisation that favours the oxidation of fatty acids in preference to carbohydrates. There was both a main effect for diet and genotype with whole-body oxygen uptake $\left(\dot{V} \mathrm{O}_{2}\right)$, in that $\dot{V} \mathrm{O}_{2}$ was elevated in response to the high-fat diet and Illo-KO BMT mice had slightly, although significantly, lower $\dot{V} \mathrm{O}_{2}$ compared with wild-type BMT controls (Table 1). The reason for this difference is not clear; however, it is unlikely to be of significant biological importance in the current context as levels of obesity were identical between genotypes.

Following 12 weeks of either the chow or the high-fat diet, plasma insulin and glucose levels were determined following a $6 \mathrm{~h}$ fast. As shown in Fig. 4a,b, animals fed a high-fat diet displayed significant hyperinsulinaemia and hyperglycaemia, compared with chow-fed animals. We observed no differences in either fasting basal insulin or glucose when comparing Ill0-KO BMT with wild-type BMT animals (Fig. 4a, b). In addition, no differences were observed in $6 \mathrm{~h}$ fed and fasted plasma insulin levels (ESM Fig. 1). Animals fed the HF diet for 12 weeks displayed a marked intolerance to both glucose and insulin compared with chow-fed animals (Fig. 4c-e). However, we did not observe any differences when comparing Il10-KO BMT with wild-type BMT mice (Fig. 4c-e). Collectively, these data demonstrate that deletion of IL-10 within the haematopoietic compartment does not exacerbate high-fat-diet-induced insulin resistance.

The adipose tissue is a key site of inflammation and macrophage recruitment in obesity and we hypothesised that a lack of the anti-inflammatory cytokine IL-10 within the haematopoietic compartment would exacerbate obesityinduced inflammation. Surprisingly, we did not observe an increase in the phosphorylation status of JNK (Fig. 5a), a 
Table 1 CLAMS metabolic and food intake data. $\dot{V} \mathrm{O}_{2}$, RER, food intake and physical activity data were collected over $24 \mathrm{~h}$ at the end of the dietary period (week 12)

Data are means \pm SEM

Main effect for diet, $* p<0.05$; genotype main effect, ${ }^{\dagger} p<0.05$

\begin{tabular}{|c|c|c|c|c|}
\hline \multirow[t]{2}{*}{ Variable } & \multicolumn{2}{|l|}{ Wild-type BM } & \multicolumn{2}{|l|}{ Illo-KO BM } \\
\hline & Chow & High-fat diet & Chow & High-fat diet \\
\hline Food intake $\left(\mathrm{kJ} \mathrm{kg}^{-1} \mathrm{day}^{-1}\right)$ & $1,905 \pm 69$ & $1,255 \pm 86^{*}$ & $2,080 \pm 130$ & $1,439 \pm 139^{*}$ \\
\hline Food intake (kJ/day) & $56 \pm 2.2$ & $47.4 \pm 2.9$ & $55.1 \pm 2.2$ & $54.1 \pm 5.2$ \\
\hline$\dot{V} \mathrm{O}_{2}\left(\mathrm{ml} \mathrm{kg}^{-1} \mathrm{~h}^{-1}\right)$ & $3,874 \pm 65$ & $3,395 \pm 100^{*}$ & $3,732 \pm 99$ & $3,096 \pm 125^{*}$ \\
\hline$\dot{V} \mathrm{O}_{2}(\mathrm{ml} / \mathrm{h})$ & $114 \pm 3$ & $129 \pm 5^{\dagger}$ & $99 \pm 2$ & $116 \pm 4^{\dagger}$ \\
\hline RER & $0.89 \pm 0.010$ & $0.78 \pm 0.007^{*}$ & $0.88 \pm 0.013$ & $0.78 \pm 0.007^{*}$ \\
\hline Total movement $(x, y)$ & $18,285 \pm 1,592$ & $19,241 \pm 1,642$ & $16,990 \pm 1,642$ & $17,894 \pm 1,896$ \\
\hline
\end{tabular}

key inflammatory kinase linked to the development of insulin resistance. However, we observed an increase in the phosphorylation status of IKK $\beta$ and an increase in the mRNA expression of the pro-inflammatory genes Tnf- $\alpha$ and $i N o s$, in high-fat-diet- compared with chow-fed mice (Fig. 5b, c). Furthermore, F4/80 (also known as Emrl) mRNA expression, a marker of macrophage recruitment, and CD11c were elevated in the adipose tissue of high-fat-fed vs chow-fed mice (Fig. 5c). No significant genotype or dietary effects were observed for Il6, Il1 $\beta$, Ifn $\gamma$ (also known as Ifng) or Mgll (also known as Clec10a) mRNA expression
(Fig. 5c). We observed no differences in any of these variables when comparing Il10-KO BMT mice with wildtype BMT mice (Fig. 5a-c). In addition to the adipose tissue, the liver is also an important site of pro-inflammatory events in obesity. However, in contrast with the adipose tissue, we were unable to detect any effect of the high-fat diet on JNK or IKK $\beta$ phosphorylation, or on any of the pro-inflammatory cytokine genes, compared with chowfed mice (Fig. 5d-f). Furthermore, no genotype affect was observed on any of the variables we assessed (Fig. 5d-f). Taken together, these data indicate that while the high-fat a

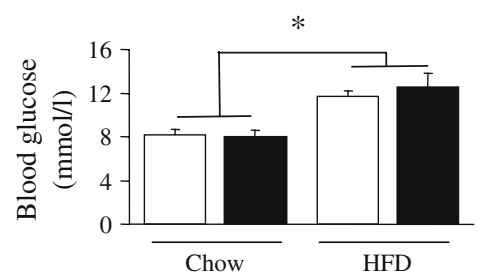

C

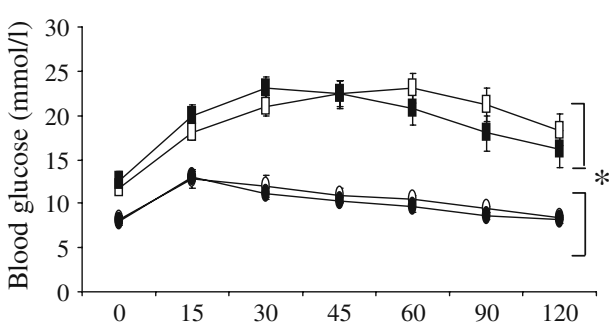

Fig. 4 Ill0-KO BMT mice have no differences in glucose homeostasis compared with wild-type BMT controls on both chow and high-fat diets. Data were collected from Ill0-KO BMT and wild-type BMT control mice after 12 weeks on chow and high-fat diets. All glucose homeostasis data were recorded after a $6 \mathrm{~h}$ fast. Levels of fasting blood glucose (a) and fasting plasma insulin (b); white bars, wild-type BMT mice; black bars, Ill0-KO BMT mice. Results from IPGTT b

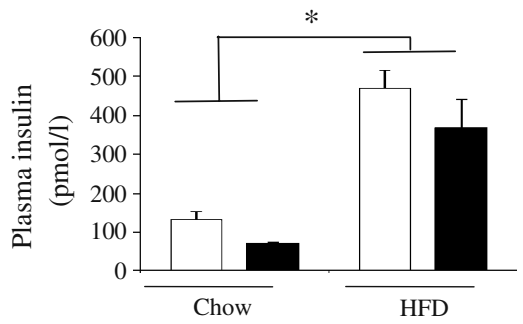

d
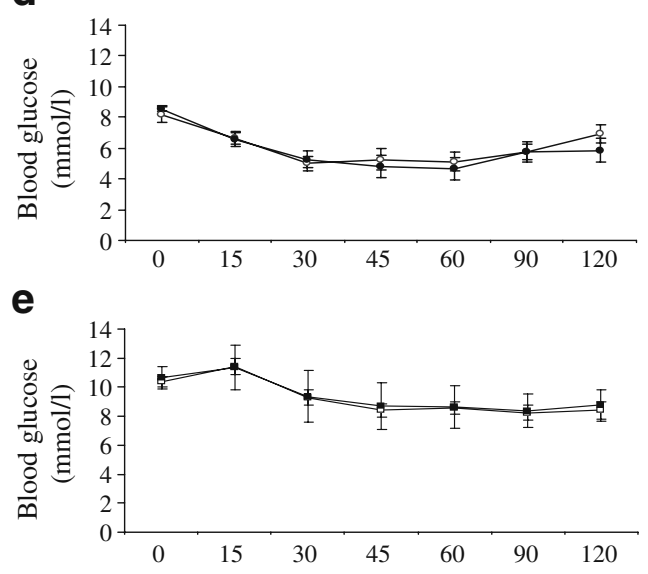

glucose $1 \mathrm{~g} / \mathrm{kg}$ LBM (c) and IPITT insulin $0.6 \mathrm{U} / \mathrm{kg}$ LBM for chowfed (d) and $0.75 \mathrm{U} / \mathrm{kg}$ LBM for high-fat-fed (e) animals. Circles, chow-fed mice; squares, high-fat-fed mice; white symbols, wild-type BMT mice; black symbols, Il10-KO BMT mice. Data are means \pm SEM. Main effect for diet, ${ }^{*} p<0.05$. Chow, $n=5-6$ per genotype; high-fat diet, $n=11-16$ per genotype 
Fig. 5 Ill0-KO BMT mice on both chow and high-fat diets have no differences in inflammatory markers in white adipose tissue $(\mathbf{a}-\mathbf{c})$ or liver $(\mathbf{d}-\mathbf{f})$ compared with wild-type BMT controls. Phosphorylation of JNK $(\mathbf{a}, \mathbf{d})$ and $\operatorname{IKK} \beta(\mathbf{b}, \mathbf{e})$ were measured using western blotting and values were expressed over the corresponding total protein values. White bars, wild-type BMT mice; black bars, Il10-KO BMT mice. c,f Cytokine gene expression as measured by RTPCR. White bars, chow-fed wild-type BMT mice; black bars, chow-fed Il10-KO BMT mice; dark grey bars, high-fatfed wild-type BMT mice; light grey bars, high-fat-fed Ill0-KO BMT mice. Data are means \pm SEM. Main effect for diet, ${ }^{*} p<$ 0.05 . Chow, $n=5-6$ per genotype; high-fat diet, $n=11-16$ per genotype. HFD, high-fat diet; P-, phosphorylated; T-, total; WT, wild type a

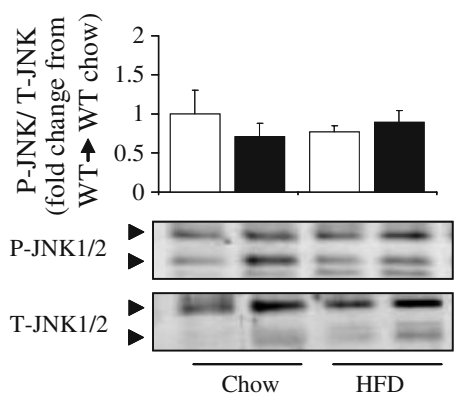

C

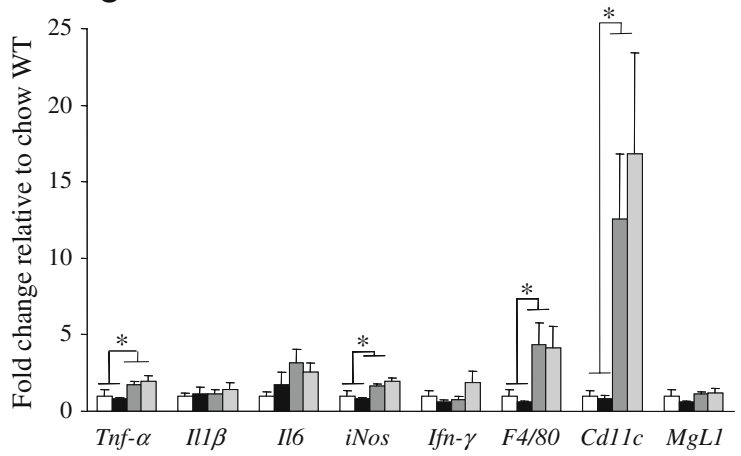

e

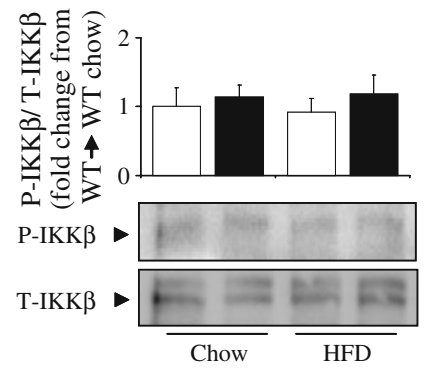

d

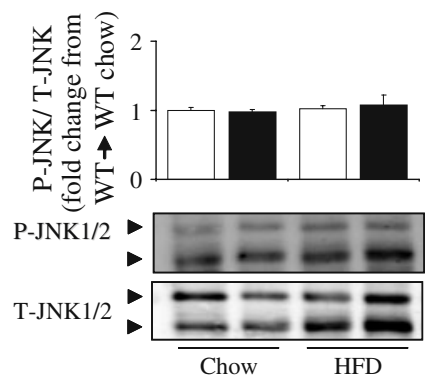

b

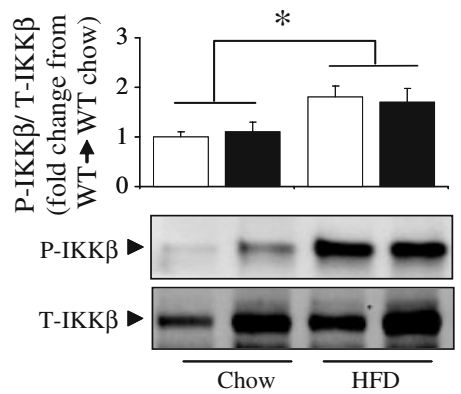

f

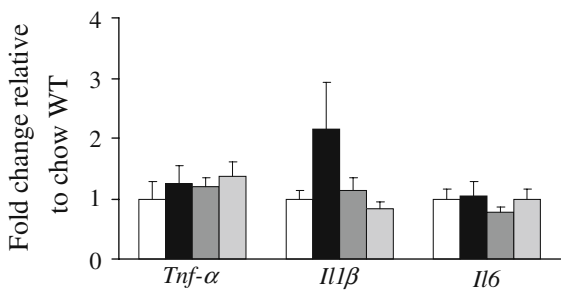

diet promoted both an inflammatory response and the recruitment of macrophages to the adipose tissue, this response was not exacerbated by the deletion of immunecell-derived IL-10.

Given the unexpected nature of these results (i.e. we did not observe a potentiation in inflammation in the Illo-KO BMT mice compared with the wild-type BMT mice), we next sought to confirm that macrophages from wild-type and Ill0-KO mice were equally able to stimulate proinflammatory responses following treatment with various inflammatory stimuli. Accordingly, we performed in vitro experiments using BMDMs harvested from Ill0-KO and wild-type mice. LPS-induced increases in the gene expression of Tnf- $\alpha$, Il6 and $I l 1 \beta$ (Fig. 6a) as well as phosphorylation of IKK $\beta$ (Fig. 6c) and JNK (Fig. 6d) and the release of TNF- $\alpha$ (ESM Fig. 2a) were nearly identical between Il10-KO and wild-type BMDMs. As expected, LPS treatment increased $I l 10 \mathrm{mRNA}$ expression and IL-10 release into the media in wild-type but not Ill0-KO
BMDMs (Fig. 6a, ESM Fig. 2b). Next we stimulated these cells with IL-4, which is known to polarise macrophages towards an 'M2' phenotype. IL-4 treatment increased Arg1 mRNA expression to similar levels in Il10-KO and wild-type BMDMs (Fig. 6b). Finally, in an attempt to mimic the action of the high-fat diet in vivo, we stimulated BMDMs with the long-chain saturated fatty acid palmitate. Palmitate treatment increased both the phosphorylation of JNK (Fig. 6e) and TNF- $\alpha$ release into the media (ESM Fig. $2 c$ ), but consistent with results observed for LPS stimulation, no differences were observed when comparing $1 / 10-\mathrm{KO}$ and wild-type BMDMs (Fig. 6e, ESM Fig. 2c).

Unexpectedly, Ill0 mRNA expression in the adipose tissue and liver of animals receiving $1 / 10-\mathrm{KO}$ bone marrow was significantly elevated compared with animals receiving wild-type bone marrow (Fig. 7a, b). However, plasma IL-10 levels were not different between wild-type BMT and Illo-KO BMT mice, and were unaffected by diet (Fig. 7c). To explore this unexpected finding in more 
a

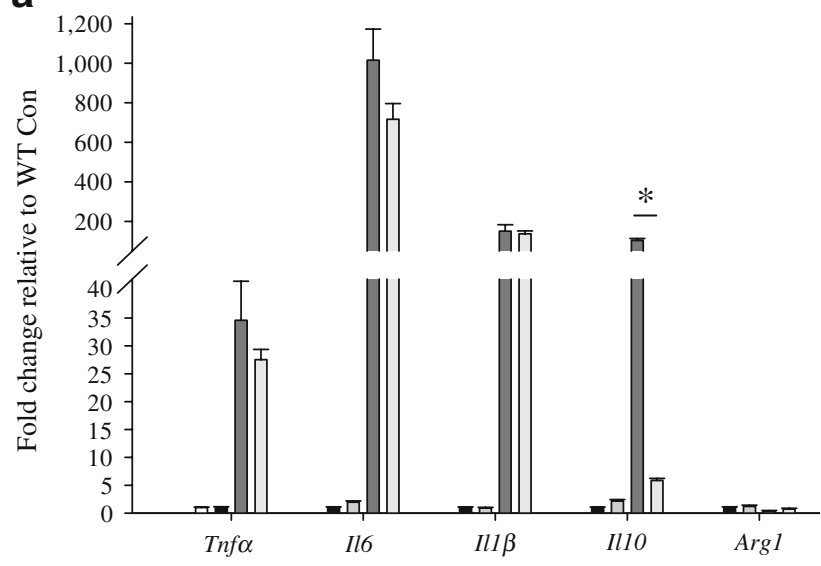

b

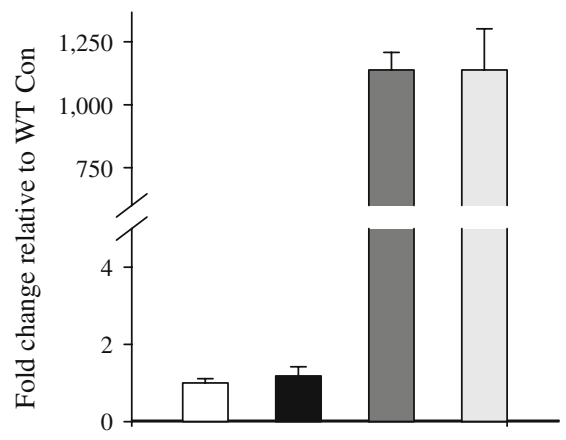

e

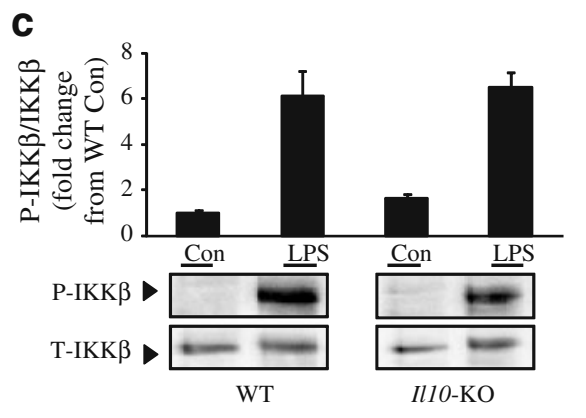

d

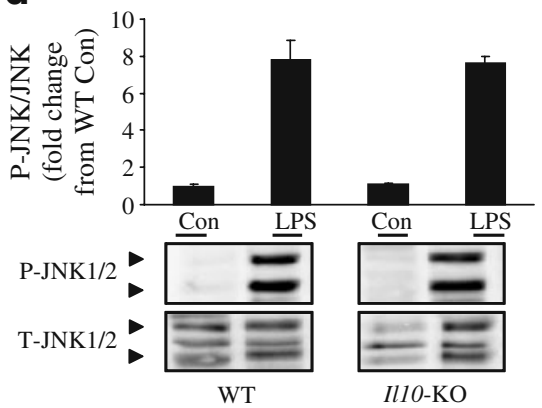

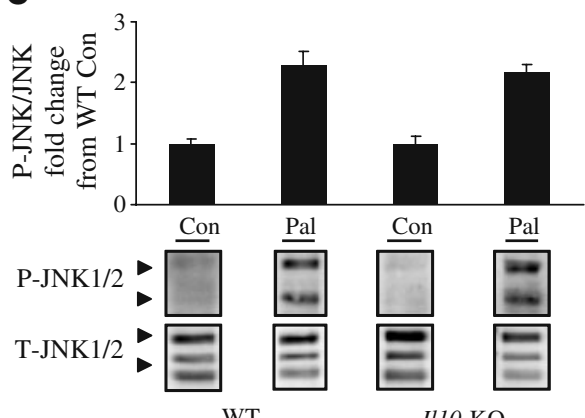

Fig. 6 BMDMs from wild-type and global $1 / 10-\mathrm{KO}$ mice have the same responses to inflammatory and immunomodulatory stimuli. a Cytokine gene expression as measured by RT-PCR from wild-type and Ill0-KO BMDMs stimulated for $6 \mathrm{~h}$ with LPS $(100 \mathrm{ng} / \mathrm{ml})$. b Argl expression in wild-type and Il10-KO BMDMs stimulated for $6 \mathrm{~h}$ with IL-4 (20 ng/ml). a,b White bars, wild-type control; black bars, Il10-KO control; dark grey bars, wild-type LPS (a) or IL-4 (b); light grey bars, Il10-KO LPS (a) or IL-4 (b). c, d Phosphorylation of IKK $\beta$ and JNK in response to $30 \mathrm{~min}$ LPS $(100 \mathrm{ng} / \mathrm{ml})$ stimulation in BMDMs from wild-type and Ill0-KO mice. e Phosphorylation of JNK in response to $4 \mathrm{~h}$ of $0.5 \mathrm{mmol} / \mathrm{l}$ palmitate treatment. Phosphorylation was measured by western blotting and values were expressed over the corresponding total protein values. Results are from three independent experiments performed in triplicate. Data are means \pm SEM. Main effect for genotype, ${ }^{*} p<0.05$. Con, control; P-, phosphorylated; Pal, palmitate treated; T-, total; WT, wild type detail we examined IL-10 protein production in adipose tissue by immunohistochemistry. IL-10 production (as indicated by brown staining) in the adipose tissue was markedly more abundant in the sections obtained from Il10-KO BMT adipose tissue compared with wild-type BMT adipose tissue (Fig. 8). The specificity of the IL-10 staining was confirmed using adipose tissue from global Ill0-KO mice (ESM Fig. 3).

\section{Discussion}

To address the role IL-10 derived from bone marrow cells (haematopoietic) plays in high-fat-diet-induced obesity, metabolic tissue inflammation and insulin resistance, we have used an adoptive transfer model whereby we transplanted bone marrow from global $1 l 10-\mathrm{KO}$ or wild-type control mice into lethally irradiated wild-type C57BL/6 recipient mice, thus creating chimeras that can produce IL-10 in all cells except those that are derived from the haematopoietic (immune cell) compartment. We hypothesised that deletion of IL-10 from the immune cell compartment would exacerbate high-fat-diet-induced inflammation in key metabolic tissues and, therefore, lead to a potentiation of high-fat-diet-induced insulin resistance. Unexpectedly, however, IL-10 deletion did not potentiate high-fat-diet-induced adipose tissue or liver inflammation, nor did it affect the severity of high-fat-diet-induced insulin resistance, suggesting endogenous immune-cell-derived IL-10 may not play a critical role in maintaining metabolic homeostasis in vivo.

In the present study we used a high-fat diet which contained $42 \%$ of energy from fat. This 12 week dietary intervention resulted in marked obesity, insulin resistance and inflammation as evidenced by the significant increases in Tnf- $\alpha$, iNos, F4/80 and Cd11c (also known as Itgax) mRNA expression and IKK $\beta$ phosphorylation in 
a

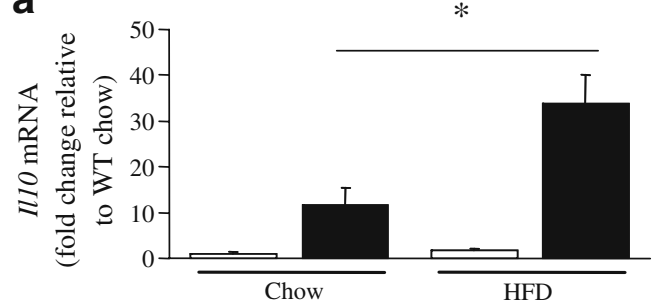

b

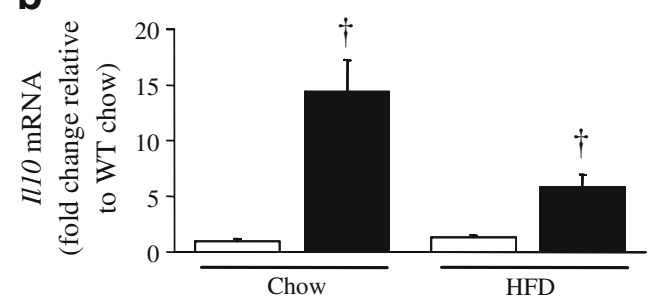

C

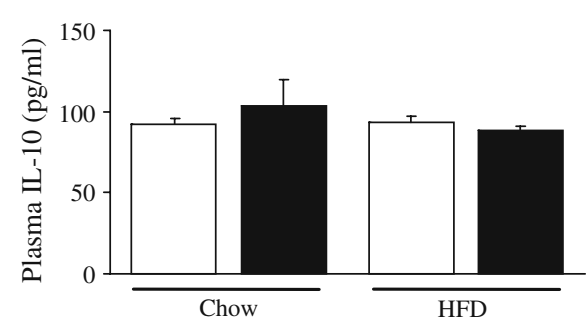

Fig. 7 Ill0-KO BMT mice have increased $1 l 10$ gene expression in white adipose tissue and liver compared with wild-type BMT controls, yet have unaltered plasma IL-10 levels. Il10 gene expression in white adipose tissue (a) and liver (b) as measured by RT-PCR. c Plasma IL-10 levels as measured by ELISA. Data are means \pm SEM. Main effect for genotype, ${ }^{*} p<0.05$; high-fat diet lower than chow-fed group, diet $\times$ genotype interaction effect, ${ }^{\dagger} p<0.05$. White, wild-type BMT mice; black, Ill0-KO BMT mice. Chow, $n=5-6$ per genotype; high-fat diet, $n=11-16$ per genotype. HFD, high-fat diet

adipose tissue. The degree of obesity ( $\sim 40 \mathrm{~g}$ body weight on high-fat diet) and white adipose tissue inflammation (Tnf- $\alpha$, iNos, F4/80, Cd11c gene induction) in this study was remarkably similar to that seen in other studies using BMT mice, even under conditions of longer-term high-fatdiet feeding (16-20 weeks) using the diet in which $60 \%$ of energy is from fat $[8,9,36]$, thus demonstrating the effectiveness of the type of high-fat diet used and the duration of the feeding protocol. Despite the high-fat-dietinduced inflammation and insulin resistance, deletion of IL-10 from the immune cells did not exacerbate this proinflammatory phenotype. In contrast to many inflammatory diseases, the levels of inflammation observed in obesity are relatively low. It is possible that these relatively modest pro-inflammatory changes may not stimulate a potent counteractive anti-inflammatory response that requires the immune cells to upregulate IL-10 production. Consistent with this notion, Ill0 mRNA expression was not altered in wild-type BMT mice following the high-fat diet compared with chow-fed animals. Indeed, previous studies have reported variable effects of obesity on $I l 10$ expression [3, 4, 9, 37]. Furthermore, and in support of the idea that a high-fat diet may not upregulate a potent antiinflammatory response, while classic pro-inflammatory stimuli such as LPS upregulate the production of both proand anti-inflammatory cytokines, macrophages treated with the saturated fatty acid palmitate do not secrete IL10; however, they induce a strong pro-inflammatory response characterised by heightened TNF- $\alpha$ and IL- 6 secretion [28]. Collectively, these data suggest that in conditions of obesity and lipid oversupply, IL-10 does not play an important anti-inflammatory role. If obesity does not stimulate an anti-inflammatory response, including the production of IL-10, then the deletion of IL-10 from the immune cell compartment would not be expected to exacerbate obesity-induced inflammation and insulin resistance, as we observed in the current study. However, in inflammatory diseases such as colitis, psoriasis, hepatitis $\mathrm{C}$ and rheumatoid arthritis, which induce both strong pro- and anti-inflammatory responses, IL-10 does play an important positive role in disease outcome [38].

Previous studies have demonstrated that the provision of IL-10, either via administration of recombinant IL-10 or via transgenic overexpression of $I l 10$, ameliorates insulin resistance $[11,25]$. Indeed, our own in vitro experiments demonstrate that the addition of IL-10 to palmitate-treated macrophages prevents TNF- $\alpha$ production and release. Thus, while experimentally increasing IL-10 levels improves insulin sensitivity, most likely via an attenuation of pro-inflammatory processes [25], because high-fat feeding in our hands does not change IL-10 production, then the deletion of endogenous IL-10 has little effect on the level of inflammation present in the tissues and, ultimately, whole-body insulin sensitivity.

An unexpected finding of the current study was the elevated $I l 10$ mRNA expression and IL-10 protein production in key metabolic tissues (e.g. the adipose tissue and liver) in the Il10-KO BMT mice compared with the wildtype BMT mice. While the mechanism for this effect is not clear, the data suggest that the deletion of IL-10 within immune cells promotes a compensatory increase in IL-10 production. While we are unable to precisely determine the cellular origin of this increase, it was apparent from the immunohistochemical analyses that much of the staining appeared at the junctions between adipocytes, with a pattern of lower production within the adipocytes. Although speculative, these data suggest that the compensatory IL-10 production in the Ill0-KO BMT mice may have originated from the vasculature and/or the adipocytes themselves. Importantly, however, any potential effect of this increase is likely to be constrained to the local tissue environment as plasma IL-10 levels were unaltered between Il10-KO BMT 
Fig. 8 IL-10 protein production is higher in the white adipose tissue of Il10-KO BMT mice compared with wild-type BMT mice. Immunohistochemical staining for IL-10 in white adipose tissue sections from wildtype BMT and Ill0-KO BMT mice from both chow-fed and high-fat-fed groups. Images shown are from three different animals from each genotype and dietary group (12 mice in total). The IL-10 protein is stained in brown. All images were taken with $\times 20$ magnification. HFD, high-fat diet

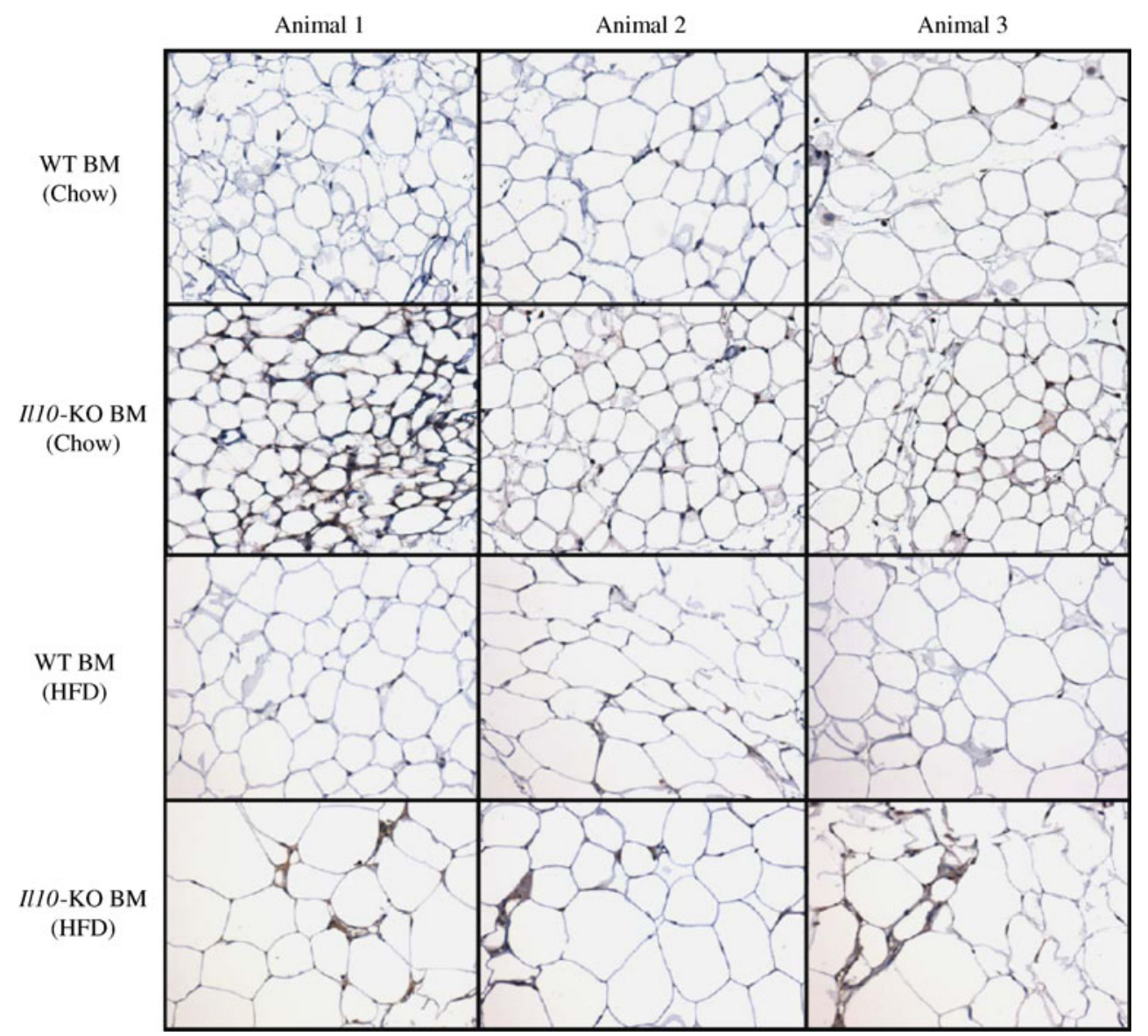

and wild-type BMT mice. It is possible that this compensatory increase in IL-10 production in the liver and adipose tissue may have suppressed pro-inflammatory responses within these tissues and, therefore, masked the effect of the deletion of IL-10 within the immune cell compartment. With regards to the cellular source contributing to the increased IL-10 production in the Ill0-KO BMT mice, the BMT technique not only largely repopulates the recipient's monocytes/macrophages with those of the donor, but also the resident liver Kupffer cells [8, 18], which are likely potential sources of liver-derived IL-10 production [29]. In addition, the BMT repopulates other bone-marrow-derived leucocytes, including lymphocytes, which are potent IL-10 producers and have also been shown to infiltrate adipose tissue and are implicated in adipose tissue inflammation and insulin resistance [39-47].

It is worth noting that, similarly to the global $I l 10-\mathrm{KO}$ mice that develop chronic enterocolitis [21, 22], a proportion (7/18) of our Il10-KO BMT mice developed mild colitis-like symptoms including loss of total body weight, loss of LBM, a failure to put on total body and fat mass with a high-fat diet, loose stools and a generally dehydrated and poor appearance. These symptoms were not observed in any of the wild-type BMT mice. These observations are consistent with several previous studies that implicate IL-10 production by lymphoid or myeloid cells in the prevention of inflammation of the large intestine and, ultimately, the development of colitis [48-50]. Given that these mice (7/18 Il10-KO BMT mice) displayed a variety of colitis-like symptoms and did not increase their fat mass during the 12 week high-fat-diet period, we excluded these mice from the study.

In summary, the results of the present study demonstrate that the deletion of IL-10 within the immune cell compartment does not exacerbate obesity-induced inflammation and insulin resistance. We hypothesise that this is due to the lack of an effect of high-fat feeding in stimulating endogenous anti-inflammatory IL-10 production, and hence deletion of IL-10 has little effect. However, our in vitro data, and results from in vivo studies of others, suggest that increasing IL-10 levels through transgenic overexpression or administration of recombinant IL-10 decreases inflammation and improves insulin sensitivity.

Acknowledgements We acknowledge the technical support provided by A. Selathurai and T. Soe Kyaw. This study was supported by 
grants from the Diabetes Australia Research Trust (to G. I. Lancaster) and the National Health and Research Council of Australia (NHMRC Project Grant No. 526619; to M. A. Febbraio and G. I. Lancaster). C. R. Bruce is an NHMRC Career Development Fellow. A. Bobik and M. A. Febbraio are NHMRC Principal Research Fellows.

Duality of interest The authors declare that there is no duality of interest associated with this manuscript.

\section{References}

1. Olefsky JM, Glass CK (2010) Macrophages, inflammation, and insulin resistance. Annu Rev Physiol 72:219-246

2. Hotamisligil GS, Shargill NS, Spiegelman BM (1993) Adipose expression of tumor necrosis factor-alpha: direct role in obesitylinked insulin resistance. Science 259:87-91

3. Weisberg SP, McCann D, Desai M, Rosenbaum M, Leibel RL, Ferrante AW Jr (2003) Obesity is associated with macrophage accumulation in adipose tissue. J Clin Invest 112:1796-1808

4. Xu H, Barnes GT, Yang Q et al (2003) Chronic inflammation in fat plays a crucial role in the development of obesity-related insulin resistance. J Clin Invest 112:1821-1830

5. Arkan MC, Hevener AL, Greten FR et al (2005) IKK-beta links inflammation to obesity-induced insulin resistance. Nat Med 11:191-198

6. Yuan M, Konstantopoulos N, Lee J et al (2001) Reversal of obesity- and diet-induced insulin resistance with salicylates or targeted disruption of Ikkbeta. Science 293:1673-1677

7. Hirosumi J, Tuncman G, Chang L et al (2002) A central role for JNK in obesity and insulin resistance. Nature 420:333-336

8. Solinas G, Vilcu C, Neels JG et al (2007) JNK1 in hematopoietically derived cells contributes to diet-induced inflammation and insulin resistance without affecting obesity. Cell Metab 6:386-397

9. Patsouris D, Li PP, Thapar D, Chapman J, Olefsky JM, Neels JG (2008) Ablation of CD11c-positive cells normalizes insulin sensitivity in obese insulin resistant animals. Cell Metab 8:301-309

10. Gordon S (2007) Macrophage heterogeneity and tissue lipids. J Clin Invest 117:89-93

11. Lumeng CN, Bodzin JL, Saltiel AR (2007) Obesity induces a phenotypic switch in adipose tissue macrophage polarization. J Clin Invest 117:175-184

12. Lumeng CN, DelProposto JB, Westcott DJ, Saltiel AR (2008) Phenotypic switching of adipose tissue macrophages with obesity is generated by spatiotemporal differences in macrophage subtypes. Diabetes 57:3239-3246

13. Lumeng CN, Deyoung SM, Bodzin JL, Saltiel AR (2007) Increased inflammatory properties of adipose tissue macrophages recruited during diet-induced obesity. Diabetes 56:16-23

14. Nguyen MT, Favelyukis S, Nguyen AK et al (2007) A subpopulation of macrophages infiltrates hypertrophic adipose tissue and is activated by free fatty acids via Toll-like receptors 2 and 4 and JNK-dependent pathways. J Biol Chem 282:35279-35292

15. Shaul ME, Bennett G, Strissel KJ, Greenberg AS, Obin MS (2010) Dynamic, M2-like remodeling phenotypes of CD11c+ adipose tissue macrophages during high fat diet-induced obesity in mice. Diabetes 59:1171-1181

16. Stienstra R, Duval C, Keshtkar S, van der Laak J, Kersten S, Muller M (2008) Peroxisome proliferator-activated receptor gamma activation promotes infiltration of alternatively activated macrophages into adipose tissue. J Biol Chem 283:22620-22627

17. Odegaard JI, Ricardo-Gonzalez RR, Goforth MH et al (2007) Macrophage-specific PPARgamma controls alternative activation and improves insulin resistance. Nature 447:1116-1120
18. Odegaard JI, Ricardo-Gonzalez RR, Red Eagle A et al (2008) Alternative M2 activation of Kupffer cells by PPARdelta ameliorates obesity-induced insulin resistance. Cell Metab $7: 496-507$

19. Gordon S (2003) Alternative activation of macrophages. Nat Rev Immunol 3:23-35

20. Saraiva M, O'Garra A (2010) The regulation of IL-10 production by immune cells. Nat Rev Immunol 10:170-181

21. Kuhn R, Lohler J, Rennick D, Rajewsky K, Muller W (1993) Interleukin-10-deficient mice develop chronic enterocolitis. Cell 75:263-274

22. Rennick D, Davidson N, Berg D (1995) Interleukin-10 gene knock-out mice: a model of chronic inflammation. Clin Immunol Immunopathol 76:S174-S178

23. Spencer SD, Di Marco F, Hooley J et al (1998) The orphan receptor CRF2-4 is an essential subunit of the interleukin 10 receptor. J Exp Med 187:571-578

24. Cintra DE, Pauli JR, Araujo EP et al (2008) Interleukin-10 is a protective factor against diet-induced insulin resistance in liver. $\mathrm{J}$ Hepatol 48:628-637

25. Hong EG, Ko HJ, Cho YR et al (2009) Interleukin-10 prevents diet-induced insulin resistance by attenuating macrophage and cytokine response in skeletal muscle. Diabetes 58:2525-2535

26. Kim HJ, Higashimori T, Park SY et al (2004) Differential effects of interleukin-6 and -10 on skeletal muscle and liver insulin action in vivo. Diabetes 53:1060-1067

27. Morari J, Torsoni AS, Anhe GF et al (2009) The role of proliferator-activated receptor gamma coactivator-1alpha in the fatty-acid-dependent transcriptional control of interleukin-10 in hepatic cells of rodents. Metabolism 59:215-223

28. Samokhvalov V, Bilan PJ, Schertzer JD, Antonescu CN, Klip A (2009) Palmitate- and lipopolysaccharide-activated macrophages evoke contrasting insulin responses in muscle cells. Am J Physiol Endocrinol Metab 296:E37-E46

29. Clementi AH, Gaudy AM, van Rooijen N, Pierce RH, Mooney RA (2009) Loss of Kupffer cells in diet-induced obesity is associated with increased hepatic steatosis, STAT3 signaling, and further decreases in insulin signaling. Biochim Biophys Acta 1792:1062-1072

30. den Boer MA, Voshol PJ, Schroder-van der Elst JP et al (2006) Endogenous interleukin-10 protects against hepatic steatosis but does not improve insulin sensitivity during high-fat feeding in mice. Endocrinology 147:4553-4558

31. Cancello R, Henegar C, Viguerie N et al (2005) Reduction of macrophage infiltration and chemoattractant gene expression changes in white adipose tissue of morbidly obese subjects after surgery-induced weight loss. Diabetes 54:2277-2286

32. Clement K, Viguerie N, Poitou C et al (2004) Weight loss regulates inflammation-related genes in white adipose tissue of obese subjects. FASEB J 18:1657-1669

33. Shi H, Kokoeva MV, Inouye K, Tzameli I, Yin H, Flier JS (2006) TLR4 links innate immunity and fatty acid-induced insulin resistance. J Clin Invest 116:3015-3025

34. Janowska-Wieczorek A, Majka M, Kijowski J et al (2001) Platelet-derived microparticles bind to hematopoietic stem/progenitor cells and enhance their engraftment. Blood 98:3143-3149

35. Senftleben U, Li ZW, Baud V, Karin M (2001) IKKbeta is essential for protecting $\mathrm{T}$ cells from TNFalpha-induced apoptosis. Immunity $14: 217-230$

36. Saberi M, Woods NB, de Luca C et al (2009) Hematopoietic cellspecific deletion of toll-like receptor 4 ameliorates hepatic and adipose tissue insulin resistance in high-fat-fed mice. Cell Metab 10:419-429

37. Li P, Lu M, Nguyen MT et al (2010) Functional heterogeneity of CD11C positive adipose tissue macrophages in diet-induced obese mice. J Biol Chem 285(20):15333-15345 
38. Mocellin S, Panelli MC, Wang E, Nagorsen D, Marincola FM (2003) The dual role of IL-10. Trends Immunol 24:36-43

39. Wu H, Ghosh S, Perrard XD et al (2007) T cell accumulation and regulated on activation, normal $\mathrm{T}$ cell expressed and secreted upregulation in adipose tissue in obesity. Circulation 115:1029-1038

40. Kintscher U, Hartge M, Hess $\mathrm{K}$ et al (2008) T-lymphocyte infiltration in visceral adipose tissue: a primary event in adipose tissue inflammation and the development of obesity-mediated insulin resistance. Arterioscler Thromb Vasc Biol 28:1304-1310

41. Rausch ME, Weisberg S, Vardhana P, Tortoriello DV (2008) Obesity in C57BL/6 J mice is characterized by adipose tissue hypoxia and cytotoxic T cell infiltration. Int J Obes (Lond) 32:451-463

42. Duffaut C, Galitzky J, Lafontan M, Bouloumie A (2009) Unexpected trafficking of immune cells within the adipose tissue during the onset of obesity. Biochem Biophys Res Commun 384:482-485

43. Rocha VZ, Folco EJ, Sukhova G et al (2008) Interferon-gamma, a Th1 cytokine, regulates fat inflammation: a role for adaptive immunity in obesity. Circ Res 103:467-476

44. Feuerer M, Herrero L, Cipolletta D et al (2009) Lean, but not obese, fat is enriched for a unique population of regulatory $\mathrm{T}$ cells that affect metabolic parameters. Nat Med 15:930-939
45. Liu J, Divoux A, Sun J et al (2009) Genetic deficiency and pharmacological stabilization of mast cells reduce diet-induced obesity and diabetes in mice. Nat Med 15:940-945

46. Nishimura S, Manabe I, Nagasaki M et al (2009) CD8+ effector T cells contribute to macrophage recruitment and adipose tissue inflammation in obesity. Nat Med 15:914-920

47. Winer S, Chan Y, Paltser G et al (2009) Normalization of obesityassociated insulin resistance through immunotherapy. Nat Med 15:921-929

48. Murai M, Turovskaya $\mathrm{O}, \mathrm{Kim} \mathrm{G}$ et al (2009) Interleukin 10 acts on regulatory $\mathrm{T}$ cells to maintain expression of the transcription factor Foxp3 and suppressive function in mice with colitis. Nat Immunol 10:1178-1184

49. Roers A, Siewe L, Strittmatter E et al (2004) T cell-specific inactivation of the interleukin 10 gene in mice results in enhanced $\mathrm{T}$ cell responses but normal innate responses to lipopolysaccharide or skin irritation. J Exp Med 200:12891297

50. Rubtsov YP, Rasmussen JP, Chi EY et al (2008) Regulatory T cellderived interleukin-10 limits inflammation at environmental interfaces. Immunity 28:546-558 